\title{
BMJ
}

\section{Excluding venous thromboembolism using point of care D-dimer tests in outpatients: a diagnostic meta-analysis}

\author{
G J Geersing, general practitioner , ${ }^{1} \mathrm{~K}$ J M Janssen, clinical epidemiologist, ${ }^{1} \mathrm{R}$ Oudega, general practitioner, \\ L Bax, clinical epidemiologist, ${ }^{1,2}$ A W Hoes, professor of clinical epidemiology and primary care, ${ }^{1}$ J B Reitsma, \\ clinical epidemiologist, ${ }^{3}$ K G M Moons, professor of clinical epidemiology ${ }^{1}$
}

\begin{tabular}{l}
\hline Julius Center for Health Sciences \\
and Primary Care, University \\
Medical Center Utrecht, Utrecht, \\
Netherlands \\
\hline${ }^{2}$ Kitasato Clinical Research Center, \\
Kitasato University, Japan \\
3Department of Clinical \\
Epidemiology and Biostatistics, \\
Academic Medical Center, \\
University of Amsterdam, \\
Amsterdam, Netherlands \\
\hline Correspondence to: G J Geersing
\end{tabular}

g.j.geersing@umcutrecht.nl

Cite this as: BMJ 2009;339:b2990 doi:10.1136/bmi.b2990

\section{ABSTRACT}

Objective To review the evidence on the diagnostic accuracy of the currently available point of care D-dimer tests for excluding venous thromboembolism.

Design Systematic review of research on the accuracy of point of care D-dimer tests, using bivariate regression to examine sources of variation and to estimate sensitivity and specificity.

Data sources Studies on the diagnostic accuracy of point of care D-dimer tests published between January 1995 and September 2008 and available in either Medline or Embase.

Review methods The analysis included studies that compared point of care D-dimer tests with predefined reference criteria for venous thromboembolism, enrolled consecutive outpatients, and allowed for construction of a $2 \times 2$ table.

Results 23 studies (total number of patients 13959 , range in mean age 38-65 years, range of venous thromboembolism prevalence $4-51 \%$ ) were included in the meta-analysis. The studies reported two qualitative point of care D-dimer tests (SimpliRED D-dimer $(n=12)$ and Clearview Simplify D-dimer $(n=7))$ and two quantitative point of care D-dimer tests (Cardiac D-dimer $(n=4)$ and Triage D-dimer $(n=2))$. Overall sensitivity ranged from 0.85 (95\% confidence interval 0.78 to 0.90 ) to 0.96 (0.91 to 0.98 ) and overall specificity from 0.48 ( 0.33 to 0.62 ) to 0.74 ( 0.69 to 0.78 ). The two quantitative tests Cardiac D-dimer and Triage D-dimer scored most favourably.

Conclusions In outpatients suspected of venous thromboembolism, point of care D-dimer tests can contribute important information and guide patient management, notably in low risk patients (that is, those patients with a low score on a clinical decision rule).

\section{INTRODUCTION}

Diagnosing patients suspected of venous thromboembolism - that is, deep venous thrombosis (DVT) or pulmonary embolism $(\mathrm{PE})$ - is a major challenge because the signs and symptoms are often mild and non-specific. ${ }^{12}$ Primary care physicians in particular, who in many countries are faced with the initial presentation of venous thromboembolism and have to decide whether to refer patients for subsequent testing or not, can have difficulty diagnosing venous thromboembolism.

D-dimer testing can be used to improve the cost effectiveness of the diagnostic process. D-dimers are degradation products of cross linked fibrin that are generated during fibrinolysis, and circulating levels are typically elevated in patients with venous thromboembolism. A negative result in a D-dimer test in combination with a low pre-test probability of venous thromboembolism (as assessed by a validated clinical decision rule) can safely rule out DVT as well as PE. .-6 $^{3}$ A large variety of laboratory D-dimer tests are currently available and their accuracy has been described extensively. ${ }^{78}$

Recently, various point of care or "near patient" $\mathrm{D}$-dimer tests have been introduced. These D-dimer tests can be performed during the consultation of a patient and results are already available within 10-15 minutes. Using these tests could avoid the need for more labour intensive and time consuming laboratory D-dimer testing. This advantage is especially useful for primary care physicians, as they often have to decide on further patient management during the consultation of a patient with suspected venous thromboembolism. In this setting, referral for conventional laboratory D-dimer testing could be either impractical owing to limited access to central laboratories or time consuming for both patient and doctor. Furthermore, venous thromboembolism is confirmed by subsequent imaging tests in only $10-20 \%$ of all referred patients. ${ }^{6}$ Application of point of care D-dimer tests could, therefore, be cost effective not only in primary care patients suspected of venous thromboembolism but also in secondary care outpatients, notably those presenting at emergency departments. Another advantage in this setting is that a test result can be obtained more rapidly than when using conventional laboratory testing.

Two systematic reviews concluded that a low clinical probability (as assessed by the clinical decision rule developed by Wells et $\mathrm{al}^{6}$ ) and a negative result on SimpliRED D-dimer (a point of care assay; Agen Biomedical, Brisbane, Australia) could safely exclude venous thromboembolism. ${ }^{910}$ There has nevertheless been much discussion about the diagnostic accuracy 
Citations reviewed $(\mathrm{n}=2022)$ :

Medline citations $(n=967)$

Embase citations ( $n=1053$ )

Personal communications $(n=2)$

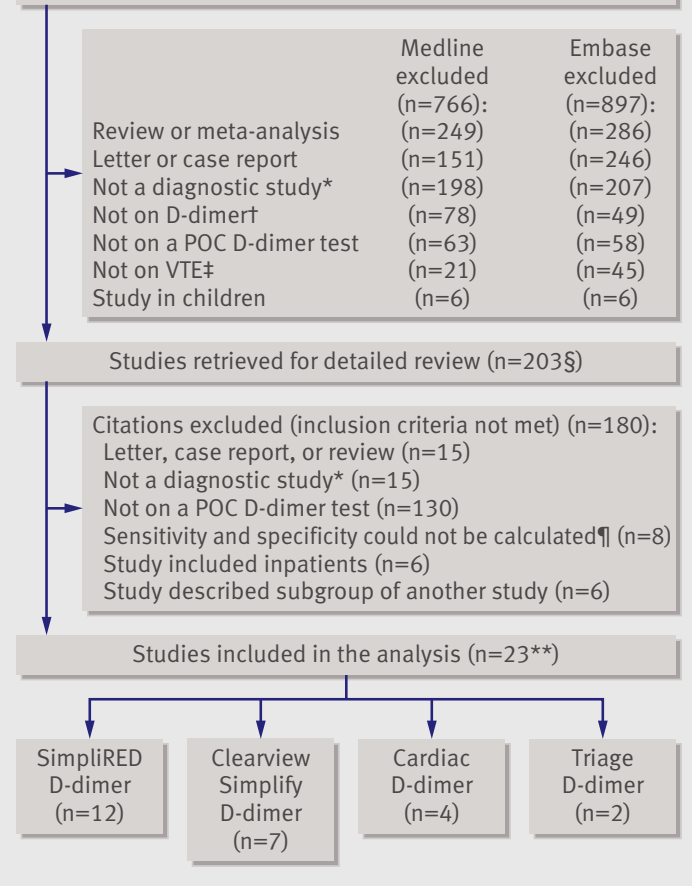

Fig 1| Flowchart of included studies. *Instead an aetiological study; for example, a study on risk factors for venous thromboembolism or number of ventilation-perfusion lung scans ordered as a function of D-dimer testing. †Studies investigating other biomarkers, such as P-selectin, or other tests, such as electrocardiography. $\ddagger$ Studies that did not use venous thromboembolism as the outcome, but aortic dissection or mesenterial thrombosis, for example. $\S 156$ of 203 studies were found in both Embase and Medline. \For example, because D-dimer testing was only performed in patients with a positive ultrasonogram. ${ }^{\star *}$ One study compared three different point of care D-dimer tests. Abbreviations: POC, point of care; VTE, venous thromboembolism

and applicability of this semiqualitative point of care D-dimer test, mainly because of the low interobserver agreement associated with this assay. ${ }^{11} 12$ Perhaps as a consequence, several new point of care D-dimer tests have been introduced in recent years. Such tests have never been subject to a formal (diagnostic) meta-analysis because studies on their diagnostic performance were not available at the time of the two latest systematic reviews. ${ }^{910}$ Before these new point of care tests are widely implemented in daily practice, however, their diagnostic performance has to be thoroughly evaluated. We conducted a diagnostic meta-analysis to assess the accuracy of all currently available point of care D-dimer tests, with particular focus on their ability to exclude either DVT or PE in suspected outpatients.

\section{METHODS}

Data sources and searches

We performed a systematic search of two online databases to identify studies evaluating the diagnostic accuracy of point of care D-dimer tests for the exclusion of DVT and PE. Sources were articles in Medline and Embase published between 1995 and 1 September 2008. The search strategy included ("fibrin fibrinogen degradation products" OR "D-dimer") combined with ("venous thrombosis" OR "pulmonary embolism". We restricted our search to studies published in English only. Duplicate articles identified in both Medline and Embase were manually deleted using Reference Manager, version 11 (Thomson Reuters, Philadelphia, PA, USA). The reference lists of identified studies were checked for further relevant studies, and experts in the field recommended further articles to complement the electronic searches.

\section{Point of care D-dimer tests under study}

D-dimers are degradation products of cross linked fibrin generated during fibrinolysis. D-dimer, however, is not a single entity in plasma but a mixture of heterogeneous fibrin degradation products. Different assays measure different types of D-dimer; consequently, test results are reported as $\mu \mathrm{g} / \mathrm{ml} \mathrm{D}$-dimer units (D-DU), $\mu \mathrm{g} / \mathrm{ml}$ fibrinogen equivalent units (FEU), or $\mathrm{ng} / \mathrm{ml}$. No single cut off value for all assays exists, and attempts to standardise D-dimer testing have failed. ${ }^{13}$ Hence, any D-dimer test can only be correctly validated against a reference standard for venous thromboembolism (for example, compression ultrasonography for DVT). We included four different point of care D-dimer tests in our meta-analysis: two qualitative tests and two quantitative tests.

\section{SimpliRED D-dimer}

SimpliRED D-dimer is a semiqualitative test performed by mixing capillary or venous blood with a drop of test reagent in the test well. A positive result is defined as any visible agglutination within two minutes. Visible agglutination will occur if D-dimer levels exceed $200 \mu \mathrm{g} / \mathrm{l}$.

\section{Clearview Simplify D-dimer}

Clearview Simplify D-dimer (Inverness Medical, Bedford, UK) is a qualitative test and is performed by mixing $35 \mu \mathrm{l}$ of capillary or venous blood with two drops of test reagent. A positive test result occurs when D-dimer levels exceed $80 \mathrm{ng} / \mathrm{ml}$ and is indicated by a visible pink-purple coloured line that forms at the test zone. Test results are valid if a pink-purple line is also present at the control zone. The test can be read within 10 minutes.

\section{Cardiac D-dimer}

Cardiac D-dimer (Roche Diagnostics, Mannheim, Germany) is performed with $150 \mu \mathrm{l}$ whole venous blood and a small portable instrument (Cobas h 232; Roche Diagnostics). The instrument displays a quantitative result within a range of $0.1-4.0 \mu \mathrm{g} / \mathrm{ml}$ FEU. A threshold value of $0.5 \mu \mathrm{g} / \mathrm{ml} \mathrm{FEU} \mathrm{is} \mathrm{used,} \mathrm{above}$ which a test result is called positive. Test results are available within 10-15 minutes. 


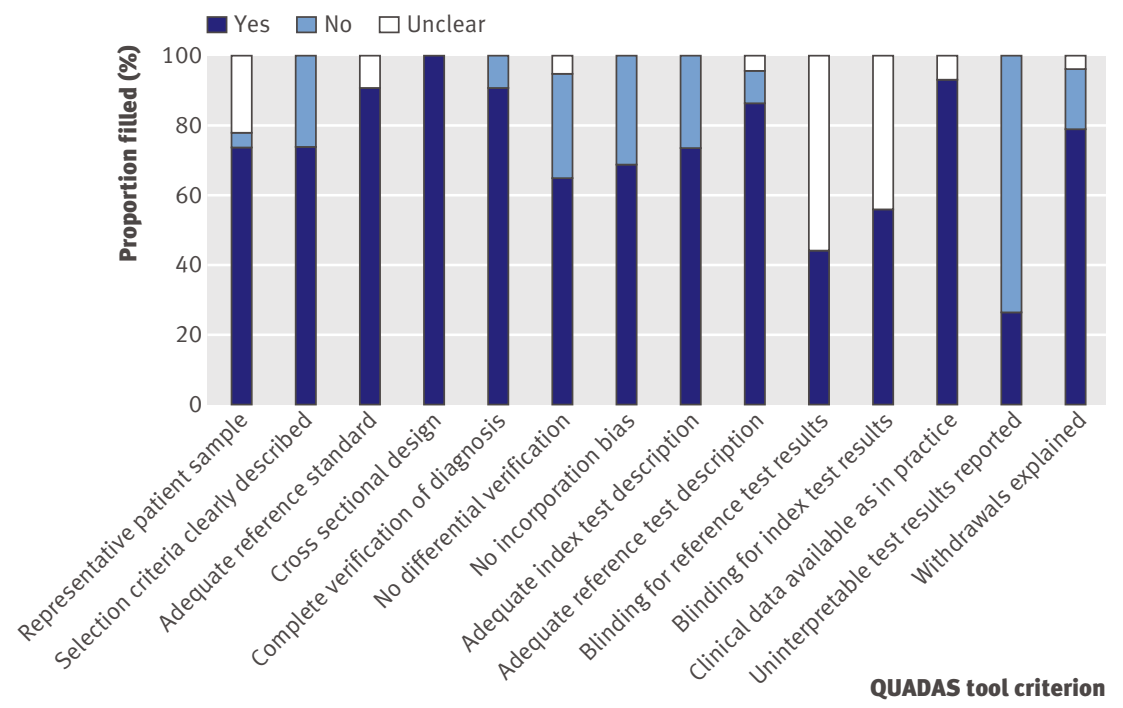

Fig 2 | Proportion of all 14 Quality Assessment of Diagnostic Accuracy Studies (QUADAS) tool criteria that were fulfilled

Triage D-dimer

Triage D-dimer (Biosite, San Diego, CA, USA) is performed with $250 \mu$ l of whole blood treated with EDTA and a small portable instrument (Triage Meter Plus; Biosite). The Triage Meter Plus displays a quantitative result within a range of $0.1-5 \mu \mathrm{g} / \mathrm{ml} \mathrm{D}-\mathrm{DU}$, using a cut off value of $0.35 \mu \mathrm{g} / \mathrm{ml}$ D-DU. Test results are available within 10-15 minutes.

\section{Study selection}

To be eligible for inclusion, the studies had to fulfil the following criteria:

1) Have a study population of consecutive outpatients (age $>18$ years) in primary or secondary care who were clinically suspected of DVT (proximal or distal) and/or PE

2) Assess a point of care D-dimer test; that is, a test that is performed in a near patient situation in the emergency department or at the physician's office and not on a routine laboratory machine

3) Use compression ultrasonography, venography, impedance plethysmography, or uneventful follow-up (that is, no DVT or PE in at least three months, an accepted reference standard in this field of research ${ }^{6}$ ) as a reference test for DVT

4) Use computerised tomography pulmonary angiography, ventilation-perfusion lung scanning, pulmonary angiography, or uneventful follow-up as a reference test for $\mathrm{PE}$

5) Include calculation of sensitivity, specificity, negative and positive predictive values, and prevalence of DVT or PE.

\section{Data extraction}

Two reviewers (GJG and KJMJ) independently extracted the following characteristics from each study: prevalence of DVT and PE; percentage males; percentage of patients with an active malignancy; percentage of patients with recent surgery ( $<4$ weeks); and mean age of the patients.
If the study addressed DVT, the percentage of patients with a distal (calf) vein thrombosis and the percentage of patients with a previous DVT were also extracted. Finally, the number of true positives, false positives, true negatives, and false negatives for the index test were extracted from the studies and a $2 \times 2$ table was constructed. Discrepancies were resolved by discussion between the two reviewers and if agreement could not be reached, a third or fourth reviewer was consulted (RO and KGMM). Authors of the respective studies were contacted to provide further details in cases where information was missing from the articles.

\section{Quality assessment}

The methodological quality of the selected studies was graded independently by two reviewers (GJG and KJMJ) with the Quality Assessment of Diagnostic Accuracy Studies (QUADAS) tool, a validated tool for the quality assessment of diagnostic accuracy studies. ${ }^{14}$ In case of doubt, a third and fourth reviewer (RO and KGMM) were consulted.

\section{Data synthesis and analysis}

We used the bivariate model for diagnostic meta-analysis to obtain an overall sensitivity and an overall specificity. ${ }^{15}$ Instead of using the diagnostic odds ratio, as used in conventional diagnostic meta-analytical methods, ${ }^{16}$ the bivariate approach uses pairs of sensitivity and specificity as the starting point of the analysis. Besides accounting for study size, the bivariate model estimates and incorporates the negative correlation that may arise between the sensitivity and specificity of the index test within studies as a result of differences in test positive/ negative threshold between studies. These threshold differences are particularly important for the qualitative point of care D-dimer tests, as the threshold underlying a positive result in such tests is unknown or implicit. The bivariate model uses a random effects approach for both sensitivity and specificity, which allows for heterogeneity beyond chance as a result of clinical and methodological differences between studies. To examine sources of heterogeneity, different study characteristics were added to the model as covariates. For reasons of statistical power, we performed such a meta-regression analysis for only point of care D-dimer tests assessed in at least 10 studies. For the remaining tests, we simply performed a subgroup analysis across relevant subgroups of the covariate. Evidence for publication bias through small study effects was explored with a regression test on the diagnostic odds ratio. ${ }^{17}$

We defined a priori the following clinical and design characteristics of a study as potential relevant covariates: type of venous thromboembolism (DVT or PE); proportion of patients with a proximal DVT (for DVT studies only); proportion of patients with a recurrent DVT (for DVT studies only); proportion of patients with an active malignancy; and proportion of patients with recent surgery ( $<4$ weeks). The QUADAS tool criteria, in particular differential verification and incorporation bias, were also examined as potential sources of bias. 


\section{Table 1|Population and study characteristics of included studies}

\begin{tabular}{|c|c|c|c|c|c|c|c|c|c|c|}
\hline Study & $\begin{array}{c}\text { No of } \\
\text { partici- } \\
\text { pants }\end{array}$ & $\begin{array}{l}\text { Mean age } \\
\text { (years) }\end{array}$ & $\begin{array}{c}\text { Proportion } \\
\text { males (\%) }\end{array}$ & $\begin{array}{c}\text { Proportion } \\
\text { with active } \\
\text { malignancy } \\
\text { (\%) }\end{array}$ & $\begin{array}{l}\text { Proportion } \\
\text { with recent } \\
\text { surgery (\%) }\end{array}$ & $\begin{array}{l}\text { Prevalence } \\
\text { VTE (\%) }\end{array}$ & $\begin{array}{c}\text { Prevalence } \\
\text { distal DVT (\%) } \\
\text { * }\end{array}$ & $\begin{array}{l}\text { Prevalence } \\
\text { recurrent DVT } \\
(\%)^{\star}\end{array}$ & $\begin{array}{c}\text { Proportion of } \\
\text { patients who } \\
\text { underwent } \\
\text { imaging and } \\
\text { follow-up (\%)† }\end{array}$ & $\begin{array}{c}\text { Reference tes } \\
\text { used }\end{array}$ \\
\hline \multicolumn{11}{|l|}{ DVT studies } \\
\hline \multicolumn{11}{|l|}{ SimpliRED D-dimer } \\
\hline Wells et al, $1995^{\mathrm{w} 13}$ & 214 & 56 & 37 & ? & ? & 25 & 12 & 0 & $100 / 0$ & Venography \\
\hline $\begin{array}{l}\text { Anderson et al, } \\
2000^{\text {w3 }}\end{array}$ & 214 & 54 & 44 & 5 & 5 & 13 & 0 & 0 & $100 / 0$ & $\begin{array}{l}\text { CUS and } \\
\text { venography }\end{array}$ \\
\hline $\begin{array}{l}\text { van der Graaf et al, } \\
2000^{w 12}\end{array}$ & 99 & 58 & 38 & $?$ & 0 & 51 & 12 & ? & $100 / 0$ & Venography \\
\hline Kearon et al, $2001^{\text {w8 }}$ & 443 & 60 & 36 & $?$ & $?$ & 14 & $?$ & 0 & $60 / 40$ & Combined $\ddagger$ \\
\hline Bozic et al, $2002^{\mathrm{w5}}$ & 135 & 60 & 39 & $?$ & $?$ & 38 & 19 & $?$ & $100 / 0$ & CUS \\
\hline $\begin{array}{l}\text { Kraaijenhagen et al, } \\
2002^{\mathrm{w} 11}\end{array}$ & 1695 & 60 & 37 & 13 & 15 & 22 & 0 & 0 & $100 / 0$ & CUS \\
\hline $\begin{array}{l}\text { Anderson et al, } \\
2003^{\text {w4 }}\end{array}$ & 1057 & 57 & 43 & 5 & 10 & 18 & 0 & ? & $70 / 30$ & Combined $\neq$ \\
\hline Kilroy et al, $2003^{\mathrm{w} 9}$ & 279 & ? & ? & ? & ? & 14 & ? & ? & $100 / 0$ & CUS \\
\hline \multicolumn{11}{|c|}{ Clearview Simplify D-dimer } \\
\hline Neale et al, $2004^{\mathrm{w17}}$ & 187 & $?$ & 46 & $?$ & $?$ & 27 & $?$ & $?$ & $100 / 0$ & Venography \\
\hline $\begin{array}{l}\text { Subramaniam et al, } \\
2006^{\text {w19 }}\end{array}$ & 453 & 55 & 35 & $?$ & $?$ & 19 & 63 & 0 & $100 / 0$ & CUS \\
\hline Toll et al, $2008^{\mathrm{w} 2}$ & 200 & 59 & 33 & 2 & 2 & 12 & 0 & 16 & $100 / 0$ & CUS \\
\hline $\begin{array}{l}\text { AMUSE study, } \\
2009^{w 1}\end{array}$ & 1002 & 57 & 37 & 5 & 8 & 13 & 0 & 15 & $50 / 50$ & Combined $\neq$ \\
\hline \multicolumn{11}{|l|}{ Cardiac D-dimer } \\
\hline Bucek et al, $2001^{\text {w22 }}$ & 85 & 58 & 41 & 31 & $?$ & 41 & 22 & $?$ & $100 / 0$ & $\begin{array}{l}\text { CUS and } \\
\text { venography }\end{array}$ \\
\hline $\begin{array}{l}\text { Legnani et al, } \\
2003^{\mathrm{w} 20}\end{array}$ & 80 & $21-94 \S$ & 40 & $?$ & ? & 40 & 0 & 0 & $100 / 0$ & CUS \\
\hline${ }_{1}^{\text {Dempfle et al, } 2006^{\mathrm{w} 2}}$ & 560 & 57 & 38 & 6 & 0 & 39 & 0 & 0 & $100 / 0$ & CUS \\
\hline Toll et al, $2008^{\mathrm{w} 2}$ & 200 & 59 & 33 & 2 & 2 & 12 & 0 & 16 & $100 / 0$ & CUS \\
\hline \multicolumn{11}{|l|}{ Triage D-dimer } \\
\hline Toll et al, $2008^{\text {w2 }}$ & 200 & 59 & 33 & 2 & 2 & 12 & 0 & 16 & $100 / 0$ & CUS \\
\hline \multicolumn{11}{|l|}{ PE studies } \\
\hline \multicolumn{11}{|l|}{ SimpliRED D-dimer } \\
\hline $\begin{array}{l}\text { Ginsberg et al, } \\
1998^{\mathrm{w7}}\end{array}$ & 1177 & 53 & 41 & ? & ? & 17 & NA & NA & $100 / 0$ & Other" \\
\hline Kline et al, $2001^{\mathrm{w} 10}$ & 380 & 50 & 30 & 15 & 12 & 17 & NA & NA & $100 / 0$ & Other" \\
\hline Wells et al, $2001^{\mathrm{w} 14}$ & 930 & 50 & 37 & 7 & 8 & 9 & NA & NA & $50 / 50$ & Combined $\ddagger$ \\
\hline \multicolumn{11}{|c|}{ Clearview Simplify D-dimer } \\
\hline Hogg et al, $2005^{\mathrm{w} 15}$ & 417 & 38 & 48 & 1 & 1 & 5 & NA & NA & $41 / 59$ & Combined $\neq$ \\
\hline Kline et al, $2006^{\mathrm{w} 16}$ & 2302 & 44 & 31 & 8 & 5 & 4 & NA & NA & $45 / 55$ & Combined $\ddagger$ \\
\hline Runyon et al, $2008^{\mathrm{w} 18}$ & 1169 & 47 & 34 & 5 & 5 & 4 & NA & NA & $51 / 49$ & Combined $\ddagger$ \\
\hline \multicolumn{11}{|l|}{ DVT \& PE studies } \\
\hline \multicolumn{11}{|l|}{ SimpliRED D-dimer } \\
\hline Farrell et al, $2000^{\text {w6 }}$ & 173 & 55 & 41 & $?$ & $?$ & 33 & NA & NA & $100 / 0$ & Other** \\
\hline \multicolumn{11}{|l|}{ Triage D-dimer } \\
\hline Ghys et al, $2007^{\mathrm{w} 23}$ & 308 & 65 & 55 & ? & ? & 3 & NA & NA & $45 / 55$ & Combined $\ddagger$ \\
\hline
\end{tabular}

CUS, compression ultrasonography; DVT, deep venous thrombosis; NA, not applicable; PE, pulmonary embolism; ?, not reported

*Proportion distal and recurrent DVT among all DVT patients; †Proportion of patients who underwent an imaging test as the reference test/proportion of patients who had clinical follow-up only as the reference test; $\ddagger$ Both imaging and clinical follow-up as the reference test, where patients with a low pre-test probability and a negative D-dimer were not referred for imaging but only followed up; §Only age range was given; đPatients initially underwent pulmonary imaging (ventilation-perfusion lung scan or computed tomography). In patients with a non-diagnostic ventilation-perfusion lung scan, serial CUS, pulmonary angiography , or venography was used; **All patients underwent objective imaging. Those suspected of DVT also underwent CUS, whereas those suspected of PE underwent a ventilation-perfusion lung scan, pulmonary angiography, or computed tomography scanning

To graphically present the results, we plotted the individual and summary points of sensitivity and specificity in a receiver operating characteristic graph, plotting the index test's sensitivity (true positive rate) on the $y$ axis against 1-specificity (false negative rate) on the $\mathrm{x}$ axis. In addition, we plotted the 95\% 

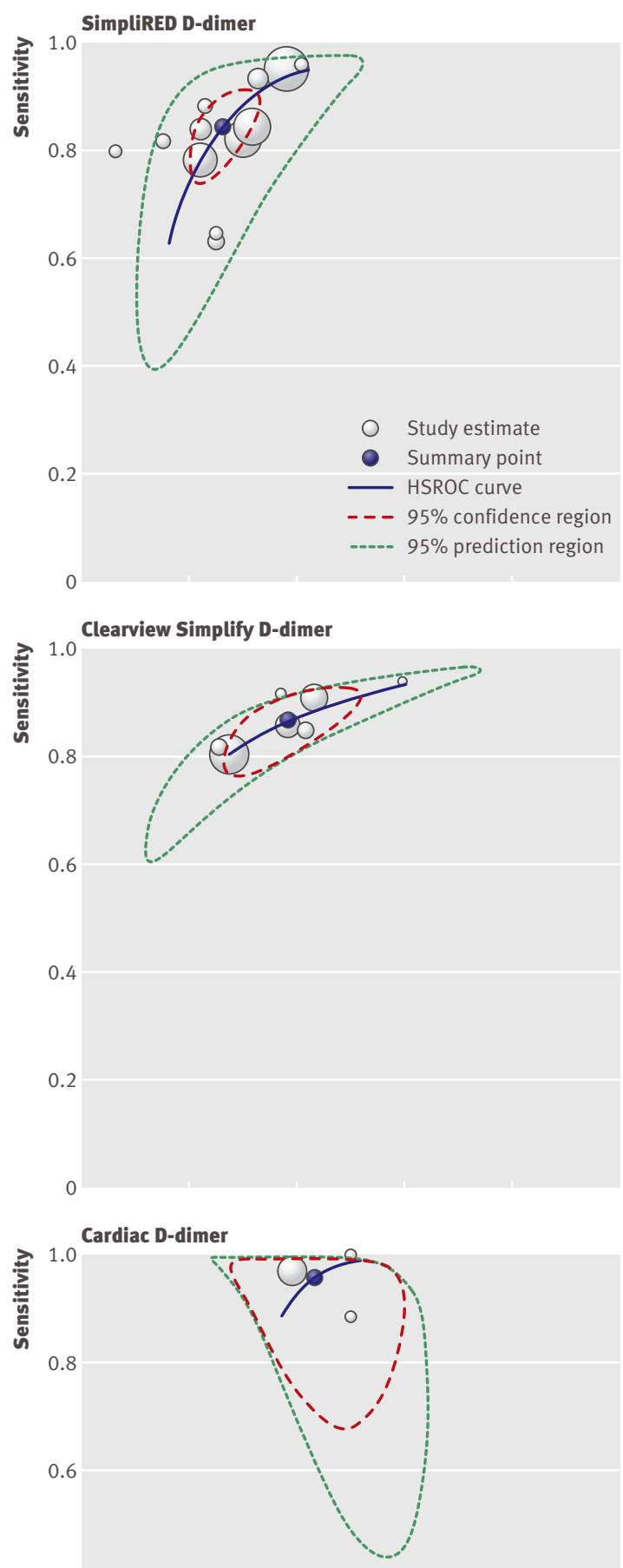

0.4

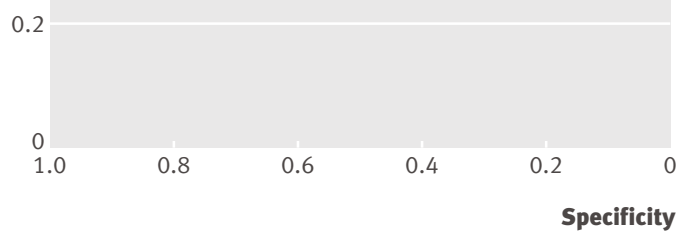

Fig 3 | Receiver operating characteristic graphs with $95 \%$ confidence region and $95 \%$ prediction region for SimpliRED, Clearview Simplify, and Cardiac D-dimer.

Abbreviation: HSROC, hierarchical summary receiver operating characteristic confidence region and a $95 \%$ prediction region around the pooled estimates to illustrate the precision with which the pooled values were estimated (confidence ellipse of a mean) and to show the amount of between study variation (prediction ellipse; the likely range of values for a new study).

Finally, we estimated the predictive value of the point of care D-dimer tests. Given that D-dimer testing is predominately used to exclude venous thromboembolism, the post-test probability of a negative D-dimer test - that is, the negative predictive valueis of highest importance. As the incidence of venous thromboembolism following normal results from imaging tests is around $2 \%$ for patients suspected of DVT, ${ }^{6}$ a post-test probability of $2 \%$ or less would be accepted as safe. According to Bayes' theorem, the post-test probability of a negative test can be calculated using the negative likelihood ratio; that is, the pre-test odds multiplied by the likelihood ratio of a negative test result (LR-), where pre-test odds is calculated by dividing pre-test probability by (1-pre-test probability) and the post-test probability comprises post-test odds divided by (1+post-test odds). ${ }^{18}$ The pooled estimates of sensitivity and specificity were used to calculate the average LR- by dividing (1-sensitivity) over specificity. Subsequently, we estimated the negative predictive value of the different point of care D-dimer tests for three different clinically relevant prevalences of venous thromboembolism: ${ }^{619}$ 5\% prevalence (low risk patients); $20 \%$ prevalence (moderate risk patients); and $50 \%$ prevalence (high risk patients). Where appropriate, 95\% confidence intervals were calculated.

We used STATA version 10.0 (StataCorp, College Station, TX, USA), in particular the midas and metandi commands, for all statistical analyses. ${ }^{2021}$

\section{RESULTS}

Identification of studies and study quality

Our search yielded 2022 citations (fig 1), 203 of which were retrieved for full text review. Of these, 180 articles were excluded, mainly because the particular D-dimer test examined was not a point of care assay. A total of 23 citations were included in the final analysis. The 23 studies included a large management study (1002 patients suspected of DVT) that has recently been accepted for publication and a recently conducted study on the accuracy of different point of care D-dimer tests. ${ }^{\text {w1 w2 }}$ The final analysis included 12 studies evaluating SimpliRED D-dimer, ${ }^{\text {w3-w14 }}$ seven looking at Clearview Simplify D-dimer, ${ }^{\text {w1 w2 w15-w19 }}$ four examining Cardiac D-dimer, ${ }^{\text {w2 }}{ }^{\text {w20-w22 }}$ and two assessing Triage D-dimer. ${ }^{\text {w2 w23 }}$

In total, we included 6796 patients tested with SimpliRED D-dimer, 5730 patients tested with Clearview Simplify D-dimer, 925 patients tested with Cardiac D-dimer, and 508 patients tested with Triage D-dimer, of which 1272/6796 (18.7\%), 470/5730 $(8.2 \%), 317 / 925(33.9 \%)$, and $67 / 508(13.2 \%)$ were diagnosed with venous thromboembolism, respectively. Mean age of included patients varied across 
Table 2 | Overall and covariate analysis for point of care D-dimer tests

\begin{tabular}{|c|c|c|c|}
\hline & $\begin{array}{l}\text { Number of } \\
\text { studies }\end{array}$ & Sensitivity $(95 \% \mathrm{Cl})$ & Specificity $(95 \% \mathrm{Cl})$ \\
\hline \multicolumn{4}{|l|}{ SimpliRED D-dimer } \\
\hline Overall analysis* & 12 & 0.85 (0.78 to 0.90$)$ & 0.74 (0.69 to 0.78$)$ \\
\hline \multicolumn{4}{|l|}{ Covariate analysis $\dagger$} \\
\hline $\begin{array}{l}\text { Only DVT (proximal and } \\
\text { distal) }\end{array}$ & 8 & $0.86(0.78$ to 0.91$)$ & 0.75 (0.68 to 0.80$)$ \\
\hline $\begin{array}{l}\text { No differential verification } \\
\text { bias }\end{array}$ & 8 & $0.84(0.75$ to 0.90$)$ & 0.75 (0.69 to 0.80$)$ \\
\hline No incorporation bias & 8 & 0.84 (0.75 to 0.90$)$ & 0.75 (0.69 to 0.80$)$ \\
\hline \multicolumn{4}{|l|}{ Clearview Simplify D-dimer } \\
\hline Overall analysis* & 7 & 0.87 (0.81 to 0.91$)$ & $0.62(0.54$ to 0.69$)$ \\
\hline \multicolumn{4}{|l|}{ Covariate analysis $\dagger$} \\
\hline $\begin{array}{l}\text { Only DVT (proximal and } \\
\text { distal) }\end{array}$ & 4 & 0.88 (0.82 to 0.93$)$ & $0.56(0.48$ to 0.64$)$ \\
\hline $\begin{array}{l}\text { No differential verification } \\
\text { bias }\end{array}$ & 4 & 0.87 (0.79 to 0.93$)$ & $0.60(0.47$ to 0.71$)$ \\
\hline No incorporation bias & 4 & 0.87 (0.79 to 0.93$)$ & $0.60(0.47$ to 0.71$)$ \\
\hline \multicolumn{4}{|l|}{ Cardiac D-dimer } \\
\hline Overall analysis* & 4 & 0.96 (0.91 to 0.98$)$ & $0.57(0.52$ to 0.62$)$ \\
\hline \multicolumn{4}{|l|}{ Triage D-dimer $\ddagger$} \\
\hline Overall analysis & 2 & 0.93 (0.88 to 0.97$)$ & $0.48(0.33$ to 0.62$)$ \\
\hline \multicolumn{4}{|c|}{$\begin{array}{l}\text { *Between study variance for logit sensitivity and logit specificity were: } 0.51 \text { and } 0.14 \text { for SimpliRED D-dimer; } \\
0.14 \text { and } 0.18 \text { for Clearview Simplify D-dimer; and } 0.15 \text { and } 0.01 \text { for Cardiac D-dimer. Between study correlation } \\
\text { for SimpliRED D-dimer, Clearview Simplify D-dimer, and Cardiac D-dimer was }-0.76,-1 \text {, and } 1 \text {, respectively. } \\
\text { tOwing to missing information on the other a priori defined covariates (see methods) in several studies and the } \\
\text { limited number of studies available, covariate analysis was only possible for the following characteristics: type } \\
\text { of venous thromboembolism; differential verification bias; and incorporation bias. } \\
\text { fFor Triage D-dimer, pooled sensitivity and specificity could not be calculated using the bivariate model owing } \\
\text { to the small number of studies available. Instead, the traditional sample size weighted approach was used. }{ }^{16} \\
\text { Abbreviations: DVT, deep vein thrombosis. }\end{array}$} \\
\hline
\end{tabular}

studies from 38 years to 65 years, and percentage males ranged from $30 \%$ to $55 \%$.

Overall, the quality of the included studies was good (fig 2). All studies used a prospective study design and enrolled consecutive outpatients suspected of venous thromboembolism. Among the possible sources of bias in diagnostic accuracy studies, differential verification and incorporation bias were present most often at $30 \%$ and $31 \%$, respectively. Also, blinding of index and reference test results was poorly reported, as was the presence of uninterpretable test results.

\section{Study and population characteristics}

Of the 12 SimpliRED D-dimer studies, eight evaluated patients suspected of DVT, three studied patients suspected of $\mathrm{PE}$, and one studied both types of patient (table 1). Of the seven Clearview Simplify D-dimer studies, four assessed patients suspected of DVT and three evaluated patients suspected of PE, whereas one of the two Triage D-dimer studies included patients suspected of DVT or PE and the other only patients suspected of DVT. All four Cardiac D-dimer studies focused on DVT only. Table 1 lists the other study and population characteristics of all 25 patient populations.

Diagnostic accuracy indices of point of care D-dimer tests Overall analysis

The pooled sensitivity and specificity of all studies combined was 0.88 (95\% confidence interval 0.83 to
0.92 ) and 0.70 (0.62 to 0.77$)$, respectively. As anticipated, the between study variability beyond what could be expected by sampling error was substantial, with an $\mathrm{I}^{2}$ of $80 \%$ for the sensitivity results and $96 \%$ for the specificity results. Analysis of small study effects, potentially a result of publication bias, yielded no significant evidence for such effects $(\mathrm{P}=0.23)$.

\section{Per test analyses}

The pooled sensitivity (based on the bivariate model) of SimpliRED, Clearview Simplify, and Cardiac D-dimer was 0.85 (95\% confidence interval 0.78 to 0.90$), 0.87$ (0.81 to 0.91$)$, and 0.96 (0.91 to 0.98$)$, respectively (fig 3, table 2); the overall specificity was 0.74 (0.69 to 0.78 ), 0.62 (0.54 to 0.69$)$, and 0.57 (0.52 to 0.62 ), respectively. As only two studies evaluated Triage D-dimer, an overall sensitivity and specificity could not be calculated using the bivariate approach. The traditional sample size weighted approach yielded an average sensitivity of 0.93 (0.88 to 0.97$)$ and specificity of 0.48 (0.33 to 0.62$).{ }^{16}$

\section{Covariate analysis}

Results from the covariate analysis are presented in table 2. Both the sensitivity and specificity of SimpliRED D-dimer and Clearview Simplify D-dimer did not vary across covariates and were essentially the same as in the overall analysis (table 2).

\section{Negative predictive value}

Table 3 shows to what extent the different point of care D-dimer assays changed the post-test probability of venous thromboembolism - that is, the incidence of venous thromboembolism following normal results from a point of care D-dimer test - for different clinically relevant pre-test probabilities. For example, if the prevalence of venous thromboembolism is high (50\%) in a suspected population, a negative Clearview Simplify D-dimer test yields a post-test probability of $18 \%$. If, however, venous thromboembolism prevalence in the population is low (5\%), a negative Clearview Simplify D-dimer test reduces this prevalence to a post-test probability of $1.1 \%$. Figure 4 can be used to estimate post-test probabilities for other pre-test probabilities.

\section{DISCUSSION}

This is the first meta-analysis of studies on the currently available point of care D-dimer tests. Measuring D-dimer levels is an important step in the diagnostic investigation of patients suspected of venous thromboembolism. A negative $\mathrm{D}$-dimer test result in combination with a low pre-test probability of venous thromboembolism is widely used to exclude the diagnosis and thus safely reduce the number of unnecessary referrals for imaging. ${ }^{10}$ A meta-analysis performed by Fancher et al in 2004 concluded that a negative result on SimpliRED D-dimer could exclude venous thromboembolism in low risk patients ${ }^{9}$; however, after 2004 various newer point of care D-dimer tests (qualitative as well as quantitative) have become available. We summarised the literature on the diagnostic accuracy of these point of care assays, including the older SimpliRED D-dimer. 
Our meta-analysis shows that the qualitative assays (SimpliRED D-dimer and Clearview Simplify D-dimer) have a lower sensitivity (that is, a higher number of false negatives) but higher specificity (that is, a lower number of false positives) than the quantitative assays (Cardiac D-dimer and Triage D-dimer). The latter seem, therefore, better suited to rule out DVT in suspected patients: they decrease the pre-test probability of venous thromboembolism more effectively. In this regard, Cardiac D-dimer outperforms Triage D-dimer, as it is able to rule out DVT both in patients with a low pre-test risk and in those with a moderate pre-test risk.

\section{Strengths and limitations}

Some methodological issues need to be considered. Firstly, we found substantial heterogeneity across the SimpliRED D-dimer studies. This variation could not be corrected for or explained by adding covariates in the bivariate meta-regression model. In the summary receiver operating characteristic graph, all but three of the 12 'sensitivity - (1-specificity)' combinations of the individual SimpliRED D-dimer studies lie on or near the receiver operating characteristic curve; therefore, studies with a higher sensitivity have a lower specificity and vice versa. This pattern is commonly attributed to differences in the threshold for test positivity used in the different studies. ${ }^{16}$ Indeed, the heterogeneity across the SimpliRED studies could well be explained by threshold differences, as defining a positive test result for this point of care D-dimer test is subjective and depends on the amount of visible agglutination seen. This assessment may have low interobserver agreement and, therefore, different thresholds for test positivity. ${ }^{1112}$ Threshold differences across diagnostic studies are implicit, so they cannot be accounted for in the analysis. Hence, calculating a common underlying sensitivity and specificity of SimpliRED D-dimer might not be possible with the currently available data. The use of quantitative point of care assays and further standardising the interpretation of qualitative point of care tests will surely reduce this problem.

Secondly, most of the studies in our review included patients suspected of DVT. Only six studies evaluated patients suspected of $\mathrm{PE}$. In patients with $\mathrm{PE}$, there is a strong correlation between embolus location and Ddimer levels. Small sub-segmental thrombi result in less D-dimer formation, so D-dimer tests have lower sensitivity in patients suspected of PE. ${ }^{22}$ Indeed, the two of the three Clearview Simplify D-dimer studies that included patients suspected of PE had lower sensitivity than the studies that only included patients suspected of DVT. ${ }^{\text {w15 } 16}$ No studies on the diagnostic accuracy of the Cardiac D-dimer for patients suspected of PE were available; therefore, our results for patients suspected of PE should be interpreted with more caution than those relating to patients with DVT.

Thirdly, different studies used different reference standards to diagnose or exclude venous thromboembolism. As with many reference standards, none was a 24 carat gold standard. For example, compression ultrasonography is used as the reference standard

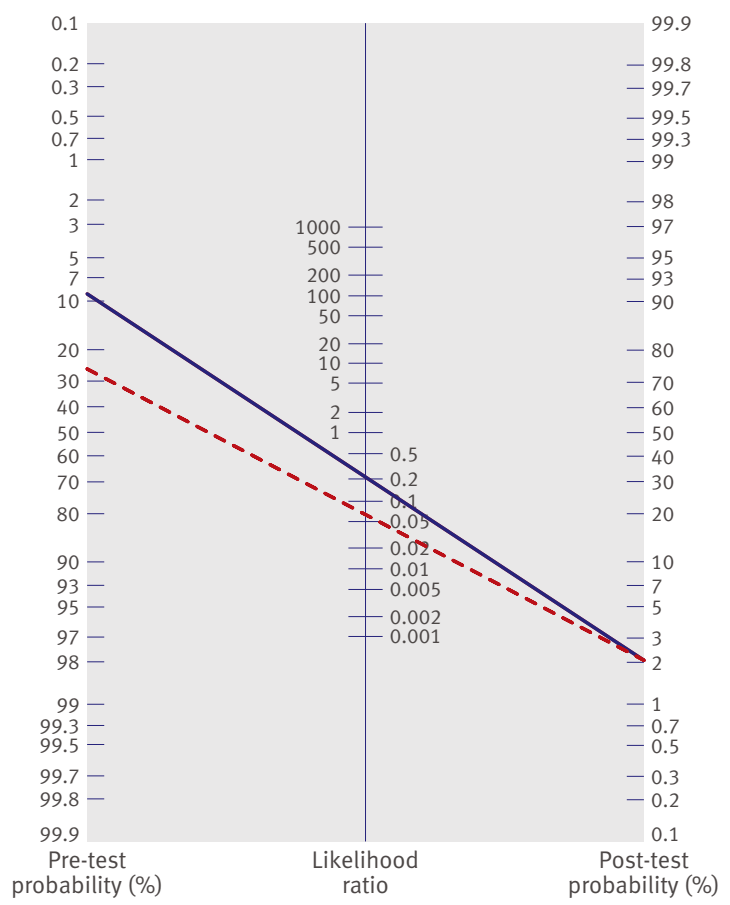

Fig 4 | Fagan's nomogram for Cardiac D-dimer (dashed red line) and Clearview Simplify D-dimer (solid blue line), with a fixed post-test probability of $2 \%$. If post-test probability is fixed at the accepted safety standard of $2 \%$, Cardiac D-dimer can exclude venous thromboembolism in patients with a pre-test probability of up to $27 \%$, compared with $9 \%$ with Clearview Simplify D-dimer. For other pre-test probabilities, the posttest probability can be calculated by simply drawing a line through the negative likelihood ratio of the respective point of care D-dimer test and the chosen pre-test probability

in most DVT studies. A meta-analysis by Goodacre et al found a pooled sensitivity of compression ultrasonography for detection of proximal DVT of 0.94 , compared with venography as reference standard. ${ }^{23}$ This finding implies that even compression ultrasonography might miss a small proportion of DVT cases. Given that most of the DVT studies in our analysis used compression ultrasonography as the reference standard, the reported pooled sensitivity of the point of care D-dimer tests could thus be slightly overestimated. Compression ultrasonography is now the accepted reference standard in daily clinical practice, however, and the use of a reference standard with a higher sensitivity - such as venography - is no longer advocated in guidelines. Also, many studies use uneventful follow-up to exclude venous thromboembolism, a common approach in this field of research. The inclusion in our analysis of studies that used uneventful follow-up could lead to a small overestimation of the sensitivity of point of care D-dimer tests, as patients with negative test results but mild DVT and/or PE could be missed if they were managed with uneventful follow-up only.

Fourthly, we restricted our search to studies published in English only, which could have been a potential source of bias. We complemented our electronic 
searches with recommendations from experts in the field, however, and this approach retrieved no non-English studies. In addition, non-English studies often have little effect on summary estimates in meta-analyses. ${ }^{24}$

Fifthly, although the quality of included studies was good in general, several studies had differential verification bias and/or incorporation bias $(30 \%$ and $31 \%$, respectively). The former refers to bias introduced by the use of different reference standards for positive and negative test results. For example, patients with a positive point of care $\mathrm{D}$-dimer test result undergo invasive imaging as reference standard, whereas patients with negative point of care D-dimer tests only receive follow-up or less invasive imaging tests as reference standard. Incorporation bias occurs when the index test is also part of the reference standard. For example, venous thromboembolism is excluded in patients with a low probability ventilation-perfusion scan only if the point of care D-dimer (index test) is negative. Lijmer et al have demonstrated that both forms of bias can affect (often overestimate) the diagnostic performance of an index test. ${ }^{25}$ However, adding both forms of bias into a covariate analysis did not change the overall sensitivity and specificity of SimpliRED D-dimer and Clearview Simplify D-dimer. A covariate analysis was not possible for Cardiac D-dimer and Triage D-dimer owing to the limited amount of studies available on these tests; however, studies on these tests (notably those on Cardiac D-dimer) did not have differential verification and/or incorporation bias.

Table $3 \mid$ Analysis of the negative predictive value of point of care D-dimer tests for patients at low, moderate, and high risk of VTE

\begin{tabular}{|c|c|c|}
\hline & $\begin{array}{l}\text { Likelihood ratio of a negative test } \\
\text { result }(95 \% \mathrm{Cl})\end{array}$ & $\begin{array}{c}\text { Post-test probability of a negative } \\
\text { test result }(95 \% \mathrm{Cl})\end{array}$ \\
\hline SimpliRED D-dimer & $0.21(0.15 \text { to } 0.29)^{\star}$ & \\
\hline Low risk & & $1.1 \%(0.8$ to 1.5$)$ \\
\hline Moderate risk & & $4.9 \%$ (3.6 to 6.8$)$ \\
\hline High risk & & $17.4 \%$ (13.0 to 22.5$)$ \\
\hline Clearview Simplify D-dimer & $0.22(0.17 \text { to } 0.28)^{*}$ & \\
\hline Low risk & & $1.1 \%(0.9$ to 1.5$)$ \\
\hline Moderate risk & & $5.2 \%(4.1$ to 6.5$)$ \\
\hline High risk & & $18.0 \%$ (14.5 to 21.9$)$ \\
\hline Cardiac D-dimer & $0.07(0.04 \text { to } 0.16)^{\star}$ & \\
\hline Low risk & & $0.4 \%(0.2$ to 0.8$)$ \\
\hline Moderate risk & & $1.7 \%$ (1.0 to 3.8$)$ \\
\hline High risk & & $6.5 \%$ (3.8 to 13.7$)$ \\
\hline Triage D-dimer & $0.18(0.08$ to 0.43$) \dagger$ & \\
\hline Low risk & & $0.9 \%(0.4$ to 2.2$)$ \\
\hline Moderate risk & & $4.3 \%(2.0$ to 9.7$)$ \\
\hline High risk & & $15.3 \%(7.4$ to 30.1$)$ \\
\hline \multicolumn{3}{|c|}{$\begin{array}{l}\text { VTE, venous thromboembolism. } \\
\text { Low risk }=5 \% \text {, moderate risk }=20 \% \text {; and high risk }=50 \% \text {. } \\
\text { *The likelihood ratio of a negative test result is calculated by dividing (1-sensitivity) over specificity, using the } \\
\text { pooled estimates from the bivariate model. } \\
\text { †For Triage } D \text {-dimer, the likelihood ratio of a negative test result is calculated using the sample size weighted } \\
\text { sensitivity and specificity. }\end{array}$} \\
\hline
\end{tabular}

Finally, only two studies were performed on Triage D-dimer and only four were performed on Cardiac D-dimer, thus limiting the precision of our results for these tests.

\section{Implications}

The laboratory based enzyme linked immunosorbent assay (ELISA) is currently advocated in the literature as the best D-dimer test for excluding venous thromboembolism in a hospital setting. Stein and colleagues report in their meta-analysis that ELISA for D-dimer has an overall sensitivity of 0.96 for DVT and 0.95 for PE. ${ }^{8}$ We found in our meta-analysis that the quantitative point of care $\mathrm{D}$-dimer tests (Cardiac D-dimer and Triage D-dimer) indeed have similar sensitivity to ELISA for D-dimer. According to Stein and colleagues, the overall sensitivity of laboratory based latex D-dimer tests is 0.85 and 0.89 for DVT and PE, respectively, ${ }^{8}$ which is equal to the average accuracy of the Clearview Simplify point of care D-dimer test.

Physicians, especially those in primary care, are confronted with a wide spectrum of patients suspected of venous thromboembolism. This spectrum could range from a low risk patient-for example, a 32 year old female on oral contraceptives with minor swelling of the leg — to a high risk patient—such as a 78 year old male with a disseminated malignancy, acute onset of dyspnoea with pain on inspiration, and swelling of the entire leg. However, patients in daily practice are hardly ever "textbook patients." It is not uncommon for the low risk patient to have DVT and the high risk patient to be diagnosed with another disease, such as heart failure. Hence, it is difficult to make a decision on which patients are to be referred for reference testing (imaging) on the basis of signs and symptoms only. Moreover, this decision often has to be made at the point of care; that is, during the consultation of the patient. One possibility is referring all patients for imaging, but this approach has a poor yield of confirmed cases of venous thromboembolism (the prevalence of venous thromboembolism has even decreased below $10 \%$ in recent years ${ }^{26}$ ) and thus seems not very cost effective. Physicians in daily practice are, therefore, in need of simple and safe additional tests, such as point of care D-dimer tests, to guide them in their diagnostic decision making.

According to our analysis, using a diagnostic strategy that includes point of care D-dimer testing to rule out venous thromboembolism without the need for imaging could achieve a post-test probability of $2 \%$ or less. This probability threshold is generally accepted as safe because the incidence of venous thromboembolism following normal imaging tests, such as compression ultrasonography in patients suspected of DVT, is also around 1-2\%. ${ }^{6}$ Applying this "safety threshold" to our results indicates that all point of care $\mathrm{D}$-dimer tests are sufficiently safe in low risk patients, as they achieved a post-test probability of $0.4-1.1 \%$ (table 3 and fig 4 ). Cardiac D-dimer seems to be the only test with the capacity to exclude DVT in patients with a moderate pre-test probability (up to $27 \%$; fig 4), although only 


\section{WHAT IS ALREADY KNOWN ON THIS TOPIC}

Several meta-analyses have shown that laboratory based D-dimer testing-such as enzyme linked immunosorbent assay — can be used to exclude venous thromboembolism

Excluding venous thromboembolism commonly requires referring a patient to a central laboratory facility

Point of care D-dimer tests are available that could enable exclusion of venous thromboembolism in a near patient situation; however, their diagnostic accuracy is largely unknown

\section{WHAT THIS PAPER ADDS}

Point of care D-dimer tests can safely exclude venous thromboembolism in low risk outpatients

Such tests can, therefore, contribute important information at the point of care and guide patient management

four studies on Cardiac D-dimer were available and thus the $95 \%$ confidence interval exceeds $2 \%$.

The decision on which point of care D-dimer test to use depends on the setting. In primary care, the Clearview Simplify D-dimer test has the advantage that it can be performed with capillary blood and thus does not require any other equipment. This makes it possible to have a test result even when making a house call, although at the expense of a lower sensitivity than the quantitative tests. This user friendliness is less important in an emergency department setting, where Cardiac D-dimer and Triage D-dimer may be preferable. These two tests might be a better option not only because of their superior accuracy, but also because other cardiovascular biomarkers-such as brain natriuretic peptide, troponin, and $\mathrm{C}$ reactive protein - can be measured with the same instrument.

\section{Conclusions}

Both qualitative and quantitative point of care $\mathrm{D}$-dimer tests can safely exclude venous thromboembolism in low risk outpatients. Quantitative tests seem to perform better than qualitative tests, although the quantity of available data is still limited. Also, there are only few studies of these tests in patients suspected of PE. In outpatients suspected of DVT, however, point of care D-dimer tests can contribute important information and guide patient management.

Contributors: GJG, KJMJ, and RO participated in study concept and design, acquisition of data, analysis and interpretation of data, and drafting of the manuscript. JBR and LB participated in study concept and design and analysis and interpretation of data, and provided statistical expertise and critical revision of the manuscript. KGMM and AWH participated in study concept and design, analysis and interpretation of data, critical revision of the manuscript, and study supervision.

Funding: Financial support was provided by the Netherlands Heart Foundation (project number 2006B237) and "Zilveren Kruis Achmea" (project number Z195). These organisations had no influence on any aspect of this study.

Competing interests: The authors have conducted previous studies with point of care D-dimer tests and have received Clearview Simplify Ddimer, Cardiac D-dimer, and Triage D-dimer testkits free of charge for study purposes. Manufacturers, however, were in no way involved in the meta-analysis
1 Oudega R, Moons KG, Hoes AW. Limited value of patient history and physical examination in diagnosing deep vein thrombosis in primary care. Fam Pract 2005;22:86-91.

2 Sanson BJ, Lijmer JG, Mac Gillavry MR, Turkstra F, Prins MH, Buller HR, for the ANTELOPE-Study Group. Comparison of a clinical probability estimate and two clinical models in patients with suspected pulmonary embolism. Thromb Haemost 2000;83:199-203.

3 Le Gal G, Righini M, Roy PM, Sanchez O, Aujesky D, Bounameaux H, et al. Prediction of pulmonary embolism in the emergency department: the revised Geneva score. Ann Intern Med 2006;144:165-71.

4 Oudega R, Moons KG, Hoes AW. Ruling out deep venous thrombosis in primary care. A simple diagnostic algorithm including D-dimer testing. Thromb Haemost 2005;94:200-5.

5 van Belle A, Buller HR, Huisman MV, Huisman PM, Kaasjager K, Kamphuisen PW, et al. Effectiveness of managing suspected pulmonary embolism using an algorithm combining clinical probability, D-dimer testing, and computed tomography. JAMA 2006;295:172-9.

6 Wells PS, Owen C, Doucette S, Fergusson D, Tran H. Does this patient have deep vein thrombosis? JAMA 2006;295:199-207.

7 Heim SW, Schectman JM, Siadaty MS, Philbrick JT. D-dimer testing for deep venous thrombosis: a metaanalysis. Clin Chem 2004;50:1136-47

8 Stein PD, Hull RD, Patel KC, Olson RE, Ghali WA, Brant R, et al. Ddimer for the exclusion of acute venous thrombosis and pulmonary embolism: a systematic review. Ann Intern Med 2004;140:589-602.

9 Fancher TL, White RH, Kravitz RL. Combined use of rapid D-dimer testing and estimation of clinical probability in the diagnosis of deep vein thrombosis: systematic review. BMJ 2004;329:821.

10 Ten Cate-Hoek AJ, Prins MH. Management studies using a combination of D-dimer test result and clinical probability to rule out venous thromboembolism: a systematic review. / Thromb Haemost 2005;3:2465-70.

11 Kraaijenhagen RA, Wallis J, Koopman MM, de Groot MR, Piovella F, Prandoni $P$, et al. Can causes of false-normal D-dimer test [SimpliRED] results be identified? Thromb Res 2003;111:155-8.

12 Perzanowski C, Eiger G. Limited interobserver agreement in the SimpliRED D-dimer assay. J Thromb Haemost 2003;1:849-50.

13 Meijer $P$, Kluft $C$. The harmonization of quantitative test results of different D-dimer methods. Semin Vasc Med 2005;5:321-7.

14 Whiting P, Rutjes AW, Reitsma JB, Bossuyt PM, Kleijnen J. The development of QUADAS: a tool for the quality assessment of studies of diagnostic accuracy included in systematic reviews. BMC Med Res Methodol 2003;3:25.

15 Reitsma JB, Glas AS, Rutjes AW, Scholten RJ, Bossuyt PM, Zwinderman AH. Bivariate analysis of sensitivity and specificity produces informative summary measures in diagnostic reviews. $J$ Clin Epidemiol 2005;58:982-90.

16 Moses LE, Shapiro D, Littenberg B. Combining independent studies of a diagnostic test into a summary ROC curve: data-analytic approaches and some additional considerations. Stat Med 1993;12:1293-316.

17 Dinnes J, Deeks J, Kirby J, Roderick P. A methodological review of how heterogeneity has been examined in systematic reviews of diagnostic test accuracy. Health Technol Assess 2005;9:1-113.

18 Sackett DL, Haynes RB, Guyatt GH, Tugwell P. Clinical epidemiology. A basic science for clinical medicine. Little, Brown and Co, 1991.

19 Chunilal SD, Eikelboom JW, Attia J, Miniati M, Panju AA, Simel DL, et al. Does this patient have pulmonary embolism? JAMA 2003;290:2849-58

20 Dwamena BA. Midas: computational and graphical routines for meta-analytical integration of diagnostic accuracy studies in Stata. Division of Nuclear Medicine, Department of Radiology, University of Michigan Medical School, Ann Arbor, Michigan, 2007.

21 Harbord RM. Metandi: Stata module for meta-analysis of diagnostic accuracy. Statistical Software Components. Boston College, Departement of Economics, 2008.

22 De Monye W, Sanson BJ, Mac Gillavry MR, Pattynama PM, Buller HR, van den Berg-Huysmans AA, et al. Embolus location affects the sensitivity of a rapid quantitative D-dimer assay in the diagnosis of pulmonary embolism. Am J Respir Crit Care Med 2002;165:345-8.

23 Goodacre S, Sampson F, Thomas S, van BE, Sutton A. Systematic review and meta-analysis of the diagnostic accuracy of ultrasonography for deep vein thrombosis. BMC Med Imaging 2005;5:6.

24 Juni P, Holenstein F, Sterne J, Bartlett C, Egger M. Direction and impact of language bias in meta-analyses of controlled trials: empirical study. Int J Epidemiol 2002;31:115-23.

25 Lijmer JG, Mol BW, Heisterkamp S, Bonsel GJ, Prins MH, van der Meulen JH, et al. Empirical evidence of design-related bias in studies of diagnostic tests. JAMA 1999;282:1061-6.

26 Le Gal G, Bounameaux H. Diagnosing pulmonary embolism: running after the decreasing prevalence of cases among suspected patients. J Thromb Haemost 2004;2:1244-6.

Accepted: 28 April 2009 